\title{
Finite-temperature scalar fields and the cosmological constant in an Einstein universe
}

\author{
M. B. Altaie ${ }^{1 *}$ and M.R. Setare $e^{2,3,4 \dagger}$ \\ ${ }^{1}$ Department of Physics, Yarmouk university, 21163 Irbid-Jordan \\ ${ }^{2}$ Institute for Theoretical Physics and Mathematics, Tehran, Iran \\ ${ }^{3}$ Department of Science, Physics group, Kordestan University, Sanandeg, Iran \\ ${ }^{4}$ Department of Physics, Sharif University of Technology, Tehran, Iran
}

(December 2002)

\begin{abstract}
We study the back reaction effect of massless minimally couupled scalar field at finite temperatures in the background of Einstein universe. Substituting for the vacuum expectation value of the components of the energy-momentum tensor on the RHS of the Einstein equation, we deduce a relationship between the radius of the universe and its temperature. This relationship exhibit a maximum temperature, below the Planck scale, at which the system changes its behaviour drastically. The results are compared with the case of a conformally coupled field. An investigation into the values of the cosmological constant exhibit a remarkable difference between the conformally coupled case and the minimally coupled one.
\end{abstract}

\section{INTRODUCTION}

Quantum fields in curved spacetimes have been investigated by many authors (for a thorough in-depth review see [1]). The basic question was: does the zero-point energy of a quantized field act as the source of a gravitational field? The works dealing with this question started by mid 1970's when matter fields were brought into connection with spacetime curvature through the calculation of the vacuum expectation value of the energy-momentum tensor $<0\left|T_{\mu \nu}\right| 0>[2-6]$. The motivations for studying this quantity stems from the fact that $T_{\mu \nu}$ is a local quantity that can be defined at a specific spacetime point, contrary to the particle concept which is global. The energy-momentum tensor also act as a source of gravity in the Einstein field equations, therefore it is expected that $\left\langle 0\left|T_{\mu \nu}\right| 0>\right.$ can plays an important role in any attempt to model a self-consistent dynamics involving the classical gravitational field coupled to the quantized matter fields. So, once $<0\left|T_{\mu \nu}\right| 0>$ is calculated in a specified background geometry, we can substitute it on the RHS of the Einstein field equation and demand self consistency. For models with a cosmological constant like the Einstein static model this means that

$$
R_{\mu \nu}-\frac{1}{2} g_{\mu \nu} R+g_{\mu \nu} \Lambda=-8 \pi<0\left|T_{\mu \nu}\right| 0>
$$

where $R_{\mu \nu}$ is the Ricci tensor, $g_{\mu \nu}$ is the metric tensor and $R$ is the scalar curvature.

The solution of (1) will determine the development of the spacetime in presence of the given matter field, for which $\mid 0>$ can be defined unambiguously. This is known as the "back-reaction problem". It is interesting to perform the calculation of $<0\left|T_{\mu \nu}\right| 0>$ in Friedman-Robertson-Walker (FRW) models since it is believed that the real universe is, more or less, a sophisticated form of the Friedman models. However the time-dependence of the spacetime metric generally creates unsolvable fundamental problems. One such a problem was the definition of vacuum in a timedependent background [7]; a time-dependent background is eligible for producing particles continuously, therefore, pure vacuum states in the Minkowskian sense do not exist. On the other hand, an investigation into the thermodynamics of a time-dependent systems lacks the proper definition of thermal equilibrium, which is a basic necessity for studying finite-temperature field theory in curved backgrounds [8].

\footnotetext{
${ }^{*}$ Electronic mail: maltaie@yu.edu.jo

${ }^{\dagger}$ Electronic mail: rezakord@yahoo.com
} 
During the last twenty five years numerous papers have been published dealing with quantum field theoretic calculations performed in curved spacetimes, and specifically in the Einstein universe. On basic reason fore choosing the Einstein universe is that it stands the two fundamental challenges mentioned above. Being static, the Einstein universe leaves no ambiguity in defining the vacuum both locally and globally [1]. This same feature also allows for thermal equilibrium to be defined unambiguously. Ford [2] has shown that a closed Robertson-Walker universe has the same vacuum energy and pressure as a static universe of instantaneously equal radius. Furthermore, the Einstein static metric can be related to the closed Robertson-Walker metric through the conformal transformations, further it was shown by Kennedy [8] that thermal Green functions for the static Einstein universe and the time-dependent Robertson-Walker universe are conformally related, hence deducing a (1-1) correspondence between the vacuum and the many particle states of both universes. So that, under the equilibrium condition, the thermodynamics of quantum fields in an Einstein universe of radius $a$ is equivalent to that of an instantaneously static closed Friedman-RobersonWalker (FRW) universe of equal radius $[2,5,9]$. This means that the results obtained in a closed FRW universe would be qualitatively the same as those obtained in an Einstein universe. This means that successive states of the Einstein universe can represent a working model that will include features of the FRW universe with non-zero cosmological constant if no geometrodynamical effect is assumed to exist.

The finite-temperature corrections to the vacuum energy of the universe is an important factor in defining its thermal development. Thus it is interesting to consider such calculation, and it would be of further interest to consider the back reaction of such cases on the development of the very early universe.

Altaie and Dowker [10] calculated the finite temperature corrections to the massless conformally coupled scalar field, the neutrino field and, the photon field in the background of an Einstein universe. The results of the calculation for the photon field was used to deduce a self-consistent solution for the Einstein field equation, i.e., a back-reaction problem, from which a relation between the temperature and the radius of the Einstein universe was deduced. However, this relation was not fully exploited at that time and therefore some of the thermodynamical aspects were kept unexposed.

Recently [11](hereafter will be referred to as I), we have investigated the back reaction effects of the conformally coupled scalar field and the photon fields in the background of the Einstein universe. We solved the Einstein field equation in each case and found a relation between the temperature and the radius of the universe. This relation exhibited a minimum radius below which no self-consistent solution for the Einstein field equation can be found. Also we have found that the system exhibit two different behaviors, one at very small radii where the temperature rises sharply with the radius until it reaches a maximum value, after which it decreases as the radius is increased. We called the first regime as the "Casimir regime" since the vacuum energy is dominant through it and we called the next one the "Planck regime" since the energy of the system follows a Planckian distribution.

In this paper we will consider the calculation of the back reaction effects of the minimally coupled massless scalar field at finite temperatures in the background of the Einstein static universe. The aim is to expose the thermal behavior of the system and compare the results with those of the conformally coupled case. Taking into consideration the renewed interest in the cosmological constant, we will also investigate the relationship between the values of the cosmological constant and the temperature for successive states of the Einstein universe for both the conformally coupled and the minimally coupled cases, in order to expose the different roles played by both fields during the Casimir and the Planck regimes. This consideration will shed light on the question of the decay of the cosmological constant during early stages of the universe (corresponding to small radii states of Einstein universe), such a question comes in the context of inflationary cosmology. Results shows that there is qualitative and an important difference in the behavior of the cosmological constant for both fields in the Casimir regime. Throughout this paper we use the natural units in which $G=c=\hbar=k_{B}=1$.

\section{BASIC FORMALISM}

The metric of the static Einstein universe is given by

$$
d s^{2}=d t^{2}-a^{2}\left[d \chi^{2}+\sin ^{2} \chi\left(d \theta^{2}+\sin ^{2} \theta d \phi^{2}\right)\right],
$$

where $a$ is the radius of the spatial part of the universe $S^{3}$ and, $0 \leq \chi \leq \pi, 0 \leq \theta \leq \pi$, and $0 \leq \phi \leq 2 \pi$.

We consider an Einstein universe being filled with a massless quantum gas in thermal equilibrium at temperature $T$. The total energy density of the system can be written as

$$
<T_{00}>_{t o t}=<T_{00}>_{T}+<T_{00}>_{0},
$$

where $<T_{00}>_{0}$ is the zero-temperature vacuum energy density (Casimir energy density) and $<T_{00}>_{T}$ is the corrections for finite temperatures, i.e., 


$$
<T_{00}>_{T}=\frac{1}{V} \sum_{n} \frac{d_{n} \epsilon_{n}}{\exp \beta \epsilon_{n}-1}
$$

where $\epsilon_{n}$ and $d_{n}$ are the eigen energies and degeneracies of the $n$th state, and $V=2 \pi^{2} a^{3}$ is the volume of the spatial section of the Einstein universe.

Normally $<T_{00}>_{T}$ is divided into two parts: the black body term, which is calculated by converting the summation into an integration and is denoted by $\left\langle T_{00}>_{T}^{b}\right.$, and the correction term, calculated from the remainder and denoted by $<T_{00}>_{T}^{a}$ (see $\left.[10]\right)$, so that (3) can be written as

$$
<T_{00}>_{t o t}=<T_{00}>_{T}^{b}+<T_{00}>_{T}^{a}+<T_{00}>_{0} .
$$

Experience tells us that in the limit $\xi \equiv T a \rightarrow 0$,we have (see [10])

$$
\lim _{\xi \rightarrow 0}<T_{00}>_{T}^{a}=-<T_{00}>_{T}^{b},
$$

so that at the low temperature limit we are left with the Casimir term only. Whereas in the high-temperature limit we have

$$
\lim _{\xi \rightarrow \infty}<T_{00}>_{T}^{a}=-<T_{00}>_{0}
$$

so that we are left with the black body term only. In the next section we will use these limits to obtain the value of the Casimir energy and the expression for the black body term.

To investigate the back-reaction effect of finite-temperature quantum fields on the behavior of the spacetime we should substitute for $\left\langle T_{00}\right\rangle_{t o t}$ on the RHS of the Einstein field, but this time with the cosmological constant $\Lambda$, i.e.

$$
R_{\mu \nu}-\frac{1}{2} g_{\mu \nu} R+g_{\mu \nu} \Lambda=-8 \pi<T_{\mu \nu}>_{t o t} .
$$

All the Einstein field equations for the system are satisfied due to the symmetry of the Einstein universe which is topologically described by $T \otimes S^{3}$, and due to the structure of $\left\langle T_{\mu \nu}>\right.$ in this geometry which comes to be diagonal, and is given by (see [1],p.186 )

$$
<T_{\mu}^{\nu}>=\frac{p(s)}{2 \pi^{2} a^{4}} \operatorname{diag}(1,-1 / 3,-1 / 3,-1 / 3),
$$

where $p(s)$ is a spin-dependent coefficient.

Since we are interested in the energy density, we will consider the $T_{00}$ only. In order to eliminate $\Lambda$ from (8) we multiply both sides with $g_{\mu \nu}$ and sum over $\mu$ and $\nu$, then using the fact that $T_{\mu}^{\mu}=0$ for massless fields, and for the Einstein universe $R_{00}=0, g_{00}=1$, and $R=\frac{6}{a^{2}}$, we get

$$
\frac{6}{a^{2}}=32 \pi<T_{00}>_{t o t} .
$$

Note that in the general case conformal anomalies do appear in the expression for $\left\langle T_{\mu}^{\mu}\right\rangle$, but because of the high symmetry enjoyed by the Einstein universe these anomalies do not appear and $\left\langle T_{\mu}^{\mu}\right\rangle$ is found to be traceless for massless particles.

\section{THE VACUUM ENERGY AND BACK REACTION}

The minimally coupled massless scalar field satisfies the covariant Klein-Gordon equation

$$
\square \phi=0,
$$

where $\square=\nabla_{\mu} \nabla^{\mu}$.

Eq. (11) was solved by Schrodinger [12] for the case of closed universe, the energy eigenvalues are given by

$$
\epsilon_{n}=\frac{[n(n+2)]^{1 / 2}}{a}, \quad n=0,1,2,3 \ldots
$$


and the degeneracy of each energy level is $d_{n}=(n+1)^{2}$.

For a minimally coupled massless scalar field in an Einstein universe the total energy density is therefore given by

$$
<T_{00}>_{t o t}=\frac{1}{2 \pi^{2} a^{4}} \sum_{n=0}^{\infty} \frac{(n+1)^{2}[n(n+2)]^{1 / 2}}{\exp \left([n(n+2)]^{1 / 2} / \xi\right)-1}+<T_{00}>_{0},
$$

In the low-temperature limit (or small radius) we find that

$$
\lim _{\xi \rightarrow 0}<T_{00}>_{t o t}=0
$$

which, by (5) and (7), means that the renormalized vacuum energy density for the minimally coupled scalar field at zero temperature vanish, i.e.

$$
<T_{00}>_{0}=0
$$

Indeed this result can be confirmed by applying the Abel-Plana summation formula

$$
\sum_{n=1}^{\infty} f(n)=\int_{0}^{\infty} f(x) d x-\frac{1}{2} f(0)+i \int_{0}^{\infty} \frac{f(i x)-f(-i x)}{e^{2 \pi x}-1} d x
$$

directly to the energy mode-sum.

In the high-temperature limit we deduce that

$$
<T_{00}>_{T}^{b}=\frac{\pi^{2}}{30} T^{4}+\frac{1}{12} \frac{T^{2}}{a^{2}}
$$

which is just half the value obtained for the photon field as would be expected. However in the limit of very large radius $a$ we obtain the usual value of the back body radiation term, i.e.

$$
\lim _{\xi \longrightarrow \infty}<T_{00}>_{\text {tot }}=\frac{\pi^{2}}{30} T^{4}
$$

In order to investigate the back-reaction effect of the field we substitute for $\left\langle T_{00}\right\rangle_{t o t}$ from (13) in (10) and request a self-consistent solution, we get

$$
a^{2}=\frac{8}{3 \pi} \sum_{n=0}^{\infty} \frac{(n+1)^{2}[n(n+2)]^{1 / 2}}{\exp \left([n(n+2)]^{1 / 2} / \xi\right)-1}
$$

This equation determines a relation between the temperature $T$ and the radius $a$ of the Einstein universe in presence of the minimally coupled massless scalar field. The solutions of this equation are shown in Fig. 1 in comparison with the results obtain earlier for the conformally coupled case. Here again two regimes are recognized: one corresponding to small values of $\xi$ where the temperature rises sharply reaching a maximum at $T_{\max } \approx 0.6 T_{p}=0.85 \times 10^{32} \mathrm{~K}$. Since this regime is controlled by the vacuum energy (the Casimir energy), therefore we call it the "Casimir regime". The second regime is what we call the "Planck regime", which correspond to large values of $\xi$, and in which the temperature asymptotically approaches zero for very large values of $a$.

From (10) and (18) we can calculate the background (Tolman) temperature of the universe in the limit of high temperature and large radius. This is found to be

$$
T_{b}=\left(\frac{45}{8 \pi^{3} a^{2}}\right)^{1 / 4}
$$

This is the same result we obtained for the conformally coupled case discussed in I. To get a glimpse of the meaning of this result, we may substitute for $a$ the present value of the Hubble length, i.e. $a=1.38 \times 10^{28} \mathrm{~cm}$ we obtain $T=31.556 \mathrm{~K}$. Conversely if we demand that the background temperature have the same value as the present equivalent temperature of the $\mathrm{CMB}$ radiation, i.e. $2.73 \mathrm{~K}$, then the radius of the Einstein universe should be $1.29 \times 10^{30} \mathrm{~cm}$. This is about two orders of magnitude larger than the estimated Hubble length.

The reason for the coincidence of the behavior of the minimally coupled and conformally coupled scalar fields in the Planck regimes stems from the fact that the field equations differs only by a factor of $1 / a^{2}$ which becomes arbitrarily small for large values of $a$. This means that the difference between the behaviors of the two fields can only be noticeable within the Casimir regime, and this difference will become even clearer in the next section when we consider the cosmological constant. In the massive cases this factor can be absorbed into the mass itself and consequently one can differentiate between the two field at the very early stages of the universe only, but as the radius of the universe grows large the difference between the minimally coupled scalar field and the conformally coupled one becomes undetectable. This conclusion is quite general and would apply in the case of FRW universe too. 


\section{THE COSMOLOGICAL CONSTANT}

The cosmological constant was first introduced by Einstein in order to justify the equilibrium of a static universe against its own gravitational attraction. The discovery of Hubble that the universe may be expanding led Einstein to abandon the idea of a static universe and, along with it the cosmological constant. However the Einstein static universe remained to be of interest to theoreticians since it provided a useful model to achieve better understanding of the interplay of spacetime curvature and of quantum field theoretic effects. Recent year have witnessed a resurgence of interest in the possibility that a positive cosmological constant $\Lambda$ may dominate the total energy density in the universe (for recent reviews see [14] and [15]). At a theoretical level $\Lambda$ is predicted to arise out of the zero-point quantum vacuum fluctuations of the fundamental quantum fields. Using parameters arising in the electroweak theory results in a value of the vacuum energy density $\rho_{v a c}=10^{6} \mathrm{GeV}^{4}$ which is almost $10^{53}$ times larger than the current observational upper limit on $\Lambda$ which is $10^{-47} \mathrm{GeV}^{4} \sim 10^{-29} \mathrm{gm} / \mathrm{cm}^{3}$. On the other hand the QCD vacuum is expected to generate a cosmological constant of the order of $10^{-3} \mathrm{GeV}^{4}$ which is many orders of magnitude larger than the observed value. This is known as the old cosmological constant problem. The new cosmological problem is to understand why $\rho_{\text {vac }}$ is not only small but also, as the current observations seem to indicate, is of the same order of magnitude as the present mass density of the universe.

The value of the cosmological constant for an Einstein universe seem to be trivial. It is directly related with the total energy density. However, since the energy density in an Einstein universe varies inversely with $a^{2}$ and not with $a^{3}$, new features are expected in the behavior of the cosmological constant. In what follows we are going to investigate the possible values of the cosmological constant for different radii of the Einstein universe in presence of the massless conformally coupled and minimally coupled scalar field. But since different radii of the universe corresponds to different temperature with a non-trivial relationship between the radius and the temperature as was found in sec. II of this paper, the values of the cosmological constant at different temperatures turns out to be non-trivial and is rather of some serious interest as we find a qualitative differences between the two types of fields.

Contracting the field equations in (8) we find that

$$
\Lambda=\frac{R}{4}=\frac{3}{2 a^{2}} .
$$

On the other hand the Einstein field equations reduces to

$$
-\frac{3}{a^{2}}+\Lambda=-8 \pi \rho_{t o t}
$$

and

$$
-\frac{1}{a^{2}}+\Lambda=\frac{8 \pi \rho_{t o t}}{3}
$$

where $\rho_{t o t}=<T_{0}^{0}>_{t o t}$. Solving the above two equations we obtain

$$
\Lambda=8 \pi \rho_{t o t}
$$

Here we will consider $\rho_{t o t}=\rho_{\text {vac }}+\rho_{\text {rad }}$, but in a more general case one can set $\rho_{\text {tot }}=\rho_{\text {vac }}+\rho_{\text {rad }}+\rho_{\text {matter }}$, with $\rho_{\text {rad }}$ belonging to the massless field filling the spatial part of the universe and $\rho_{\text {matter }}$ belonging to the pressureless dust that may exist. The addition of the energy density of the pressureless matter will not make any qualitative change in the results since $\rho_{\text {matter }}$ in an Einstein universe specifically behaves same as $\rho_{\text {vac }}$ and $\rho_{\text {rad }}$.

Using (21) and the results obtained in the previous section for the dependence of $T$ on $a$ we can solve for the dependence of $\Lambda$ on $T$. Fig. (2) depicts the relationship between the cosmological constant $\Lambda$ and the temperature for successive states of the Einstein universe under the effect of back reaction of the minimally coupled scalar field at finite temperatures in comparison with the conformally coupled field. It shows that the cosmological constant for the minimally coupled case decays monotonically from an infinite value all through the vacuum dominated regime until it reaches a comparatively small value at a critical temperature at which the system changes its behavior into the Planck regime. Whereas the case of conformally coupled case we notice that the value of $\Lambda$ is nearly constant throughout the Casimir regime and only start decaying in the Planck regime. From the point of view of inflationary models a large value of $\Lambda$ is needed to resolve the problem of horizon and the problem of flatness, and possibly to generate seed fluctuations for galaxy formation [15]. 


\section{DISCUSSION AND CONCLUSIONS}

One of the interesting points of this paper is the clear difference between the behavior of the conformally coupled massless scalar field and the minimally coupled scalar field as a result of the back reaction, this behavior which becomes clearer when the value of the cosmological constant is considered. However, one may feel uneasy with the energy scale that appears to be higher than the Planck scale in the case of the conformally coupled scalar field which was considered in I. In fact this is only fictituous because if we consider the collective effect of many fields then the resultant effect will bring the energy scale below the Planck energy. This can be easily checked if one solves the Einstein field equation with both the minimally coupled and the conformally coupled energies added up in the source, which confirms the conclusion in [13] that the range of validity of the quasi-classical approximation can be extended for large number of fields.

The conformal relationship between the static Einstein universe and the Robertson-Walker universe and the possibility to consider the Einstein universe of a given radius as representative of an instantaneously static Robertson-Walker universe [2] and the (1-1) correspondence between the vacuum and the many particle states of both universes as established by the work of Kennedy [8], suggests that the thermal behavior of a real closed universe is qualitatively similar to the results obtained in this work. Therefore, we feel that the calculations in the Einstein universe are useful in understanding the interplay between quantum fields and the curvature. Indeed in I our calculations showed that an Einstein universe with curvature radius of about two order of magnitude larger than the Hubble radius will have the same CMB temperature as the presently measured one. On the other hand the analysis of the most recent observations of CMB spectrum suggests that the curvature radius of the real universe is at least 50 times larger than the Hubble radius [16]. This is a point in favour of the practical relevance of the calculations.

The main findings are same as previously stated in I but with an additional point concerning the cosmological constant:

1. The thermal development of the universe is a direct consequence of the state of its global curvature.

2. Unless containing some pressureless matter the Einstein universe will be singular at zero temperature in presence of the minimally coupled massless scalar field, in contrast with the conformally coupled case where a non-zero radius was found as an effect of the back reaction of the non-zero Casimir energy. A non-zero expectation value of the vacuum energy density always implies a symmetry breaking event.

3. During the Casimir regime the universe is totally controlled by vacuum. The energy content of the universe is a function of its radius. Using the conformal relation between the static Einstein universe and the closed FRW universe [8], this result indicates that in a FRW model there would be a continuous creation of energy out of vacuum as long as the universe is expanding, a result which was confirmed by Parker long ago [17].

4. The cosmological constant arising from the minimal scalar field mostly decays during the Casimir regime, whereas the conformally coupled scalar field dominates most of the Casimir regime and part of the Planck regime. This indicates that the minimally coupled scalar field plays most of its effective role in regions of high curvatures.

Recenly it was shown by Ellis and Maartens [18] that the birth of an inflating universe from the state of a static Einstein universe containing minimally coupled scalar field and ordinary matter, is quite possible under certain conditions. Such models are shown to avoid the quantum gravity era. However, as remarked by the authors, the fine tuning problem in these models is to fix the initial radius. Therefore, the results presented in this paper may find some applications in such an approach for closed inflationary cosmologies.

[1] N. D. Birrell, and P. C. W.Davies, Quantum Fields in Curved Space (Cambridge University Press,Cambridge, England 1982).

[2] L. H. Ford, Phys. Rev. D 11, 3370 (1975) .

[3] J. S. Dowker, and R. Critchley, J. Phys. A 9, 535 (1976).

[4] J. S. Dowker, and M. B. Altaie, Phys. Rev. D 17, 417 (1978).

[5] G. Gibbons, and M. J. Perry, Proc. R. Soc. London, A 358, 467 (1978) .

[6] T. S. Bunch, and P. C. W.Davies, Proc. R. Soc. London, A 356, 569 (1977), Proc. R. Soc. London, A 357, 381 (1977), and Proc. R. Soc. London, A 360, 117 (1978).

[7] S. Fulling, Phys. Rev. D 72850 (1973).

[8] G. Kennedy, J. Phys. A11, L77 (1978).

[9] B. L. Hu, , Phys. lett. B103, 331 (1981).

[10] M. B. Altaie, and J. S. Dowker, Phys. Rev. D 18, (1978). 
[11] M. B. Altaie, Phys. Rev. D 65, 044028 (2002).

[12] E. Schrodinger, Comment. Pont. Acad. Sci., 2, 321 (1938).

[13] L. A. Kofman, V. Sahni and A. A. Starobinsky, Sov. Phys. JETP 58 1090, (1983).

[14] S. M. Carroll, The Cosmological Constant, astro-ph/0004075 v2 (2000).

[15] V. Sahni and A. Starobinsky, Int. J. Mod. Phys. D 9, 373 (2000).

[16] M. S. Turner, Making sense of the new cosmology, astro-ph/0202008 v1 (2002).

[17] L. Parker, Phys. Rev. 831057 (1969).

[18] G. F. R. Ellis and R. Maartens, Eternal inflation without quantum gravity, gr-qc/0211082 v1(2002).

\section{Figure Caption}

FIG 1. Comparison between the temperature-radius relationship for massless conformally coupled (dashed line) and the minimally coupled (solid line) scalar fields.

FIG 2. Comparison between the contributions of the massless conformally coupled (dashed line) and the minimally coupled (solid line) scalar fields to the cosmological constant in an Einstein universe at finite temperatures. 


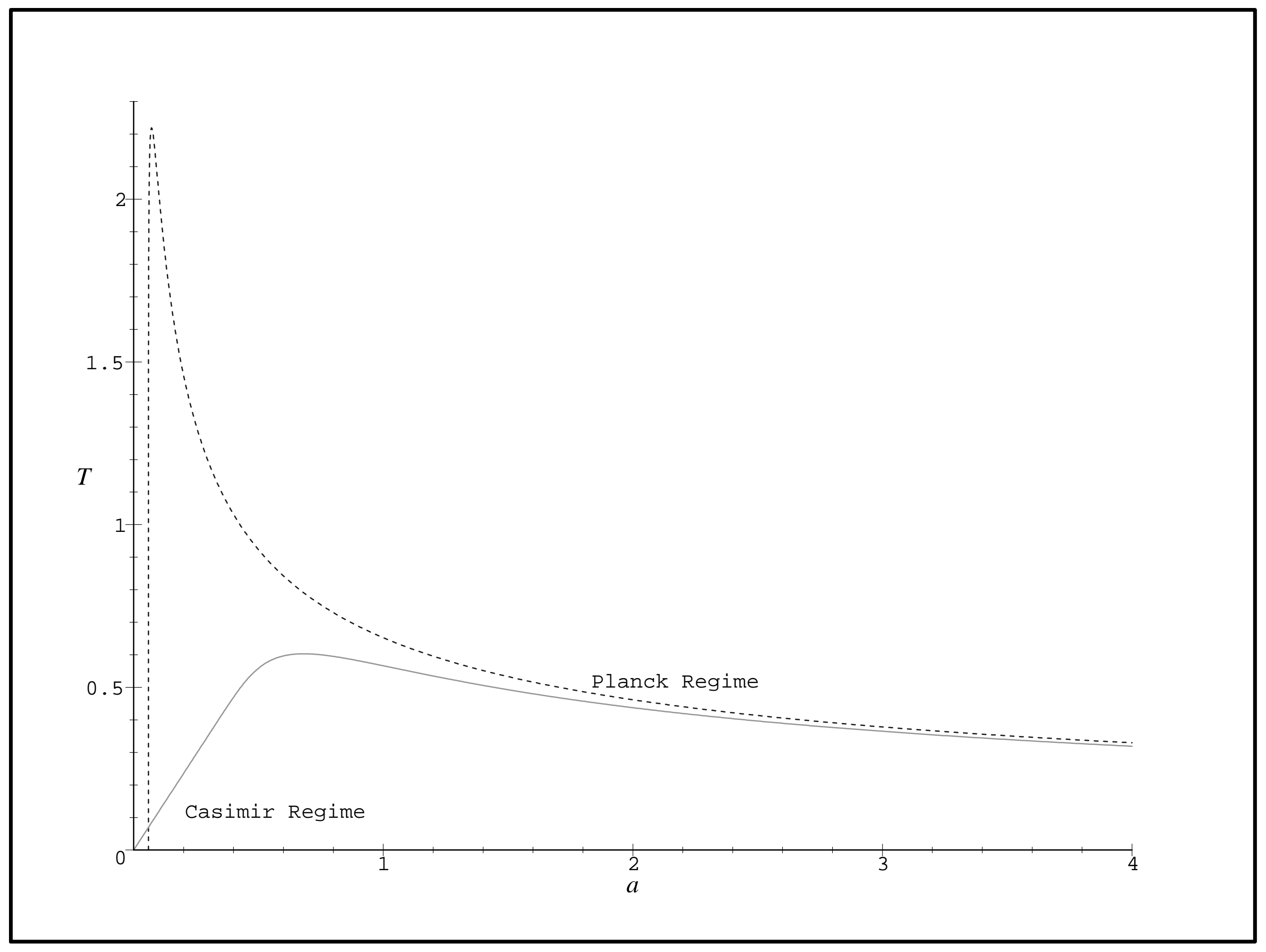




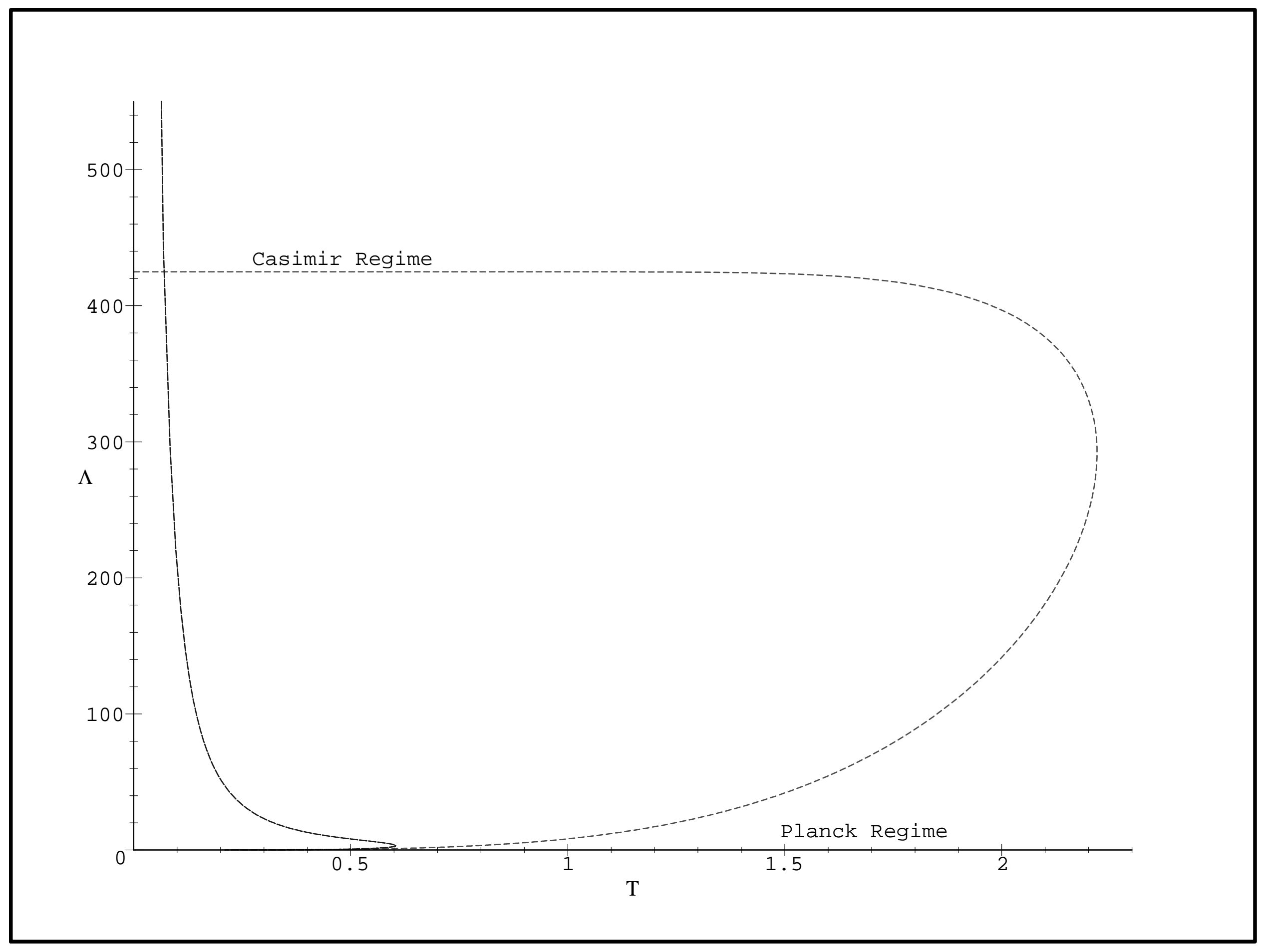

